\title{
Artificial Intelligence's Digestion and Reconstruction for Humanistic Feelings
}

\author{
Dandan Zhang, Mei Xie \\ Center for Digitalized Culture and Media \\ University of Electronic Science and Technology of China
}

\begin{abstract}
Artificial intelligence has been developed rapidly in recent years with the support and promotion of big-data technology, and we 've entered a new era -- the era of AI. In the context of more extensive application, the development of AI has brought new challenges to society and caused ethical concerns. The arrival of AI era may weaken the humanistic spirit and humanistic feelings to a certain extent, but its comprehensive application can construct a new social normal state with manmachine symbiosis, and the concept of "humanity" will also be reinterpreted.
\end{abstract}

Keywords-artificial intelligence (AI); humanistic feelings; digestion; reconstruction

\section{INTRODUCTION}

On March 9th 2016, AI robot Alpha GO, researched and developed by Google, thoroughly defeated Lee Sedol --- the world champion of Wei Qi. In early 2017, Master, the improved version of Alpha GO, defeated the master-hand, Ke Jie once again. The topic of AI has been reheated after years of silence. AI has rapid development and profound influence although with only 60 years of history since its rise. On October 11th, Sophia was awarded the citizenship of Saudi Arabia as the first robot possessing nationality and identity, which caused more worries about the application of AI. But any of new technologies has enabled mankind to reach the peak of realizing and transforming the world while bringing great impacts on human society, not excepting AI.

\section{OVERVIEW OF AI}

\section{A. The concept of AI}

The author disassembled AI into two parts for detailed comprehension: "artificial" and "intelligent". Artificial is no doubt the quintessence of human wisdom. Intelligence can be understood by its denotation as that AI can imitate human intelligence behavior and possess similar intelligence level. It should be unquestionable that the invention of AI is aimed to imitate human intelligence, alleviate stress and contribute to human society. "There are still many disputes about the judgment of "intelligent" behavior" in academic circles." [1] But it should be explicated that AI is only a helper of AI rather than a substitute.

\section{B. The classification of AI}

At present, the classification of AI has not been clearly defined in academic circles but could be summarized as three categories: Artificial Narrow Intelligence, Artificial General Intelligence and super AI.

\section{1) Artificial Narrow Intelligence}

"The definition of Artificial Narrow Intelligence was put forward at Dartmouth Conference in 1956: which means that behaviors of machines look like the intelligent behaviors of human.”[2] To summarize, Artificial Narrow Intelligence is a kind of AI specializing in one aspect such as Alpha GO, which is intelligent and powerful enough in chess but useless in other complex intellectual activities. At present, the generally contacted AI belongs to artificial narrow intelligence.

\section{2) Artificial General Intelligence}

The "strong" in the concept of Artificial General Intelligence means that AI transcends machine-type intelligence and achieves the objective and subjectival level of intelligence. Under this circumstance, "Artificial General Intelligence" is not only a tool; even the machine itself can possess thoughts and analogous abilities with human. [3] At present, the development of $\mathrm{AI}$ is in a special stage from Artificial Narrow Intelligence to Artificial General Intelligence.

\section{3) Super AI}

Ray Kurzweil believed that "in the near future, the computer will possess neopallium functions and transcend human. Human and machine will be integrated into a new object; it is predicted that the intelligence of machine will surpass human intelligence in 2045." [4] The existence of super $\mathrm{AI}$ is the main worry about AI's threat, which refers to that the Artificial General Intelligence, may exceed human's cognitive level in all fields when developed to a certain stage. It can carry out phronetal creation; possess human knowledge and even master social skills.

\section{THE APPLICATION AND INFLUENCES OF AI}

AI has been rapidly developed and attracted general attention due to its widespread application prospect despite of only 60 years of history. Its development has promoted a more convenient and quick lifestyle; and its application in various fields has facilitated new breakthroughs and developments.

\section{A. The Deep Learning Based on Big Data Technology}

The era of big data provides massive data support for the development of AI. The robot is more intelligent through a large number of simulated training and deep learning by aid of big data analysis to master a variety of complex big data 
scheme. The success of Alpha Go and its upgraded version of Master can be said as the victory of deep learning and big data to a certain extent. Every aspect of our daily life is full of AI technology, it irresistibly changes our life.

\section{B. Recognition Technology Creates Intelligent Life}

Recognition technologies e.g. voice recognition and face recognition can also help improve our work and life efficiency and ensure safety. Nowadays, face recognition can accurately identify, locate and track face information; it even enters the field of campus and office, quite a few schools and offices adopt the face recognition for attendance. The continuous breakthroughs makes identity authentication and trust delivery more simple and efficient. In addition to face recognition, there is also voice recognition. In recent years, App e.g. WeChat and Alipay have realized the function of logging by voice recognition without manual password imput. It only needs to collect user's voice and carry out contrast recognition, which also realizes the free switching between voice and text; the breakthrough of voice recognition opens a new door for the intelligent future of interpersonal interaction.

\section{The Transition of Jobs from the Old to the New}

First, the replacement crisis of jobs aggravated people's fear of unemployment due to the continuous breakthroughs in AI. According to 2016 survey data from WEF, the rise of robots and AI will lead to the loss of 5.1 million jobs in 15 major industrialized countries. Secondly, the emergence and development of each technology will lead to the decline or even disappearance of some jobs, but $\mathrm{AI}$ is also creating new jobs. However, AI has also hastened a considerable number of new types of industries and attracted a great deal of professionals and labor force due to the requirement of selfdevelopment.

\section{D. 'Machine + Journalism’ Guides New Commercial Activities of Media}

The rise of "Machine-Generated News" is the product of technological progress in the era of AI, and it is also conformed to the realistic requirements for media development in the new media era. The emergence of 'fast-pen xiao xin' of Xinhua News Agency and 'Xiao Rong' of People' s Daily new media platform have promoted AI into every link of dissemination. The application of VR in media field also manifests the deepened application of $\mathrm{AI}$ in media industry. During NPC \&CPPCC last year, Guangming Daily published the "VR Actual Scene conference of the CPPCC", which utilized VR technology to show all aspects of NPC \&CPPCC to audience. NetEase, Tencent, Sina and other Internet companies continue to launch news reports by VR technology. Robot writing makes the content production of media more efficient and deeper; VR technology makes audience's experience more real; and algorithm recommendation makes the media content accurately disseminated.

\section{AI's Digestion AgAinst Humanistic FeELINGS}

\section{A. The Aspect of Disseminator}

1) Content Production's lack of "temperature"

In the traditional media era, reporters and editors are content producers and gatekeepers. In the we-media age, everyone has microphones in their hands and socialized media enables them to become the publisher and disseminator of information. But this kind of publication and dissemination is still converted among "huaman"; however, this conversion is realized in the era of AI; and robot writing comes true. In this year, the Chinese Seismological Network sent out a message written by robot in Jiuzhaigou earthquake; it spent only 25 seconds quickly completing the whole process of data mining, data analysis and automatic writing, which was even richer and more detailed in contents. Although robot writing has unparalleled advantages in speed and depth, the news is only a pile of text, data and pictures due to its lack of thoughts and ideas, and the audience cannot feel emotions and temperatures.

\section{2) Distortion of Content Communication}

All AI's dissemination activities are based on intelligent algorithm and machine algorithm, which is both the innovation point and a weak point of AI compared to traditional technology. Algorithm serves as a computer technology to satisfy users' requirements and push content, its intelligence considers the "inaccuracy" but can't identify "correctness". It pushes messages according to users' preference but can't ensure the coincidence of content with mainstream values; and media manager's incapability of content audit and loss of values will cause serious problems. In December 2017, Beijing Internet Information Office criticized Today's Headline App for its continuous dissemination of pornographic and vulgar information and violated provision of Internet news and information services, which fully reflected the problems of algorithms and management.

\section{B. The aspect of Audience}

\section{1) Challenge of Security}

The security challenges confronted by AI greatly weakens the humanistic feelings in era of AI, which is mainly caused by immature technology development and abuse of technology. At present, $\mathrm{AI}$ is not mature enough as a developing emerging technology and easily causes technical defects, leading to serious security risks. At the same time, the effect of $\mathrm{AI}$ on human actually depends on the utilization and management mode for AI. The use of AI by criminals or evil people will bring serious security problems, e.g. the network attacks initiated by Internet hackers through intelligent methods. In May 2017, the outbreak of "worm" type extortion virus and the infection against computers of $100+$ countries and regions was exactly the outcome of abused technology. How to talk about humanistic feelings without guarantee of security?

\section{2) Challenge of Privacy Protection}

"Big data actuates the development of AI, which becomes a key feature of the new round of AI development. However, privacy becomes the greatest threat in the development and utilization of data resources."[5] The precondition of big data is to control relevant materials of users; but the privacy during 
this course is ineluctable. More serious issue lies in the use payment. Most of data grabbed through big data technology is obtained without audiences' acquirement. The correlative platforms generate products according to audiences' privacy but audiences have to pay for it.

\section{3) The Intensification of Digital Divide}

In the era of AI, audiences are not entitled to equally participate in discussions as imagined. The development of AI makes internet become a new discourse platform, however, the discourse right is still controlled by minority, with increasing difference. People with economic force and dominant right of technology occupy the upstream place of information dissemination and reception, and take Internet as their forum or platform to trumpet their viewpoints and thoughts. The general audiences who lack resources have joined in discussions, but impacts have not been made as their opinions have been ignored or defined. Secondly, the increasing aggravated gap between engineers designing $\mathrm{AI}$ with the journalists using $\mathrm{AI}$ has caused unconventional behavior in news industry.

\section{AI's ReCOnstruction fOR Humanistic FeELINGS}

\section{A. Accelerating Media Transformation}

"Compared with traditional industry, the extensive application of AI liberates physical power and part of brain work, which is no doubt characterized with humanization and humanism spirit." [6] The development of AI has contributed to the birth of robot writing, exerting great impacts on content production and journalists; but it can actually help journalists produce new news reports from another perspective, which could not be achieved in the past nor technically possible. AI has promoted the media industry's fission and transformation. It can improve journalists' work efficiency and liberate from a large number of endless repetitive work so as to devote themselves to the production of original content and depth report based on powerful AI data mining technology, which undoubtedly improves the humanistic spirit of news.

\section{B. Personalized Customization Meeting Requirements of Audiences}

Big data-based AI exerts a significant role in promoting personalized customization. AI endows the potential of customized news with stride leap and enables the articles to meet the requirements for personalized customization. Monitoring and data capture of user behavior by big data can help the journalists to understand audience preferences so as to customize news on this basis and raise their participation and loyalty. In general, major clients push varied interface to each user and are more capable of catching their preference, which is undoubtedly more humanized. And AI gradually enters our life. On the basis of mastering massive personal information, intelligent technology further improves life quality and even more understand human than us.

\section{Communication Mode Transformed from 'Monologue' to 'Dialog'}

Although the digital gap has not been reduced in AI era, dissemination mode has been transformed to a more humanized orientation. "The dissemination mode in AI era focuses on interaction with audiences and transforms from monologuemode egocentrism dissemination to interaction of dialogic interactive communication. It is transformed from monodirectional hard indoctrination to participation and bidirectional soft indoctrination. [7] Nowadays, audiences are more emotional and critical. It is also one reflection of humanism in new intelligent era to utilize dialogic dissemination mode to improve participation of audiences.

\section{CREate a New NoRmal of Society With Man- MACHINE HARMONY}

\section{A. Establishing Intelligentized Ecosystem}

With the continuous development of $\mathrm{AI}$, robots are able to own identity and the man-machine boundary becomes vaguer. The coexistence mode between human and robots is changed from "human adapt to robots" to "robots adapt to human". Robots become more similar to human, which arises considerable panics. The author, however, by now, believes the opinion that AI's replacement of human or even human's ideas only exist in science fiction films. More intelligentized machines are actually a positive consequence since it is easier to get along with than previous goofy machines. AI brings certain of impacts and challenges, but it allows us to envisage our dominant status as human; e.g. the combination of machine writing and journalists emancipates them from repetitive and monotonous work, improves efficiency, and also promotes the transformation for journalists towards original and warm creation.

\section{B. Establishing Intelligentized Management System}

At present, technology abuse becomes the primary cause of problems in development of AI. Technology is equipped with no thoughts; its functions are dominated by human. Therefore, it is urgent to establish a perfect intelligentized management system. We must realize that each new technology will cause some panics. The process of technical progress is only a process of human liberation rather than root of problems. "We could choose to live a better life humbly under the help of intelligent machine, or to die arrogantly.' [8] However, this option is controlled by the creators of AI all the time. Thus, creators and related industries should be regulated and governed; and relevant laws and regulations, as well as ethical and moral rules should be established and perfected to completely eradicate problems from the source.

\section{CONCLUSION}

In summary, the current AI can assist some complicated brain work without threats while competent for some manual labor after upgrading and improving. After all, AI is created by human who has brought it to a new height. It is only a reflection of human wisdom. "Although further developed AI is provided with abilities that exceed human intelligence, at present, it is still the shadow and extension of human organs”. [9] AI is only an artificial substitute rather than a real one. It may dispel humanization and humanism spirit to a certain extent, but it is also a reconstruction of humanistic feelings in 
the new era of intelligence. This option is always controlled by human; and we are supposed to establish related systems, strengthen management and confront the new normal state of intelligent society.

\section{REFERENCES}

[1] Xu Yingjin. Technology and Justice: Artificial Intelligence in Future War [J], Academic Pioneer, 2016(7).

[2] J. Searle .Minds Brains and Programs [J]. The Behavioral and Brain Sciences, 1980(3).

[3] Chen Jing. Technology and Ethic Move Towards Fusion-Discussion on Humanization of Artificial Intelligence [J].Academics,2017,(09)

[4] Ray Kurzweil. Near Singularity: When Humans Transcend Biology) [M]. Penguin Books,2006.
[5] Li Xiuquan. The Security, Privacy, Ethic Challenges and Solutions of Artificial Intelligence [J].Science \& Technology Review, 2017, 35(15):11-12.

[6] Du Sen. Artificial Intelligence's Influences and Its Philosophical Reflection for Industrial Economy [J]. Modern Industrial Economy and Informationization,2015(17)

[7] Lei Yang. Two Transformations of Mainstream Media Ideology Communication Mode in We-Media Era- from the Change of Popularization and Versinnlichung Trends [J].Journalism Lover, 2017(10):9-13.

[8] John Markoff. Machines of Loving Grace: The Quest for Common Ground between Humans and Robots [M]. Ecco Press,2015.

[9] Huang Xin rong. Artificial Intelligence and Human's Future [J/OL]. Journal of Xinjiang Normal University (Philosophy and Social Science Edition), 2018(04):1-8 